\title{
Questes
}

vestes Revue pluridisciplinaire d'études médiévales

$9 \mid 2006$

À la marge

\section{Sibylle au centre, sibylles à la marge}

\section{Julien Abed}

\section{OpenEdition}

\section{Journals}

Édition électronique

URL : http://journals.openedition.org/questes/2265

DOI : 10.4000/questes.2265

ISSN : 2109-9472

\section{Éditeur}

Les Amis de Questes

\section{Édition imprimée}

Date de publication : 15 juin 2006

Pagination : 52-63

ISSN : 2102-7188

\section{Référence électronique}

Julien Abed, « Sibylle au centre, sibylles à la marge », Questes [En ligne], 9 | 2006, mis en ligne le 01 janvier 2014, consulté le 03 mai 2019. URL : http://journals.openedition.org/questes/2265 ; DOI :

10.4000 /questes.2265 


\title{
Sibylle au centre, sibylles à la marge
}

\section{Lecture du Paradis de la reine Sibylle d'Antoine de La Sale}

\author{
Julien ABED
}

Dans la tradition médiévale, la sibylle a droit de cité dans la liste des femmes illustres de la mythologie antique. Façonnée par les œuvres littéraires de l'Antiquité (Virgile, Ovide), cautionnée par les Pères de l'Eglise, qui la jugent digne de figurer à côté des prophètes (Augustin, Clément d'Alexandrie), transmise par les encyclopédistes (Isidore de Séville), et consacrée par les polygraphes médiévaux, la sibylle, qui rassemble les traits de féminité, et de don prophétique, est une figure centrale pour toute la cité médiévale. Lactance, qui lègue au Moyen Age ses Institutions divines, a emprunté à un texte de Varron une liste de dix sibylles qui ont annoncé la venue du Christ ou la fin des temps : par la suite, on rencontre ces prophétesses aux frontons des églises romanes et des cathédrales gothiques, dans le texte du Dies irae, dans les sermons, les encyclopédies, dans les marges des livres d'heures, dans les œuvres littéraires, où résonnent de nombreux intertextes comme la catabase d'Enée avec la sibylle de Cumes.

Il existe aussi, en contrepoint de cette liste, à la fin du Moyen Age, une légende qui atteste l'existence d'une étrange sibylle qui peuplerait les monts Apennins : c'est la sibylle de Norcia, qui règne sur un royaume peuplé de créatures de lupanar ${ }^{1}$. Le Paradis de la reine Sibylle s'en fait l'écho. Ecrit vers

\footnotetext{
${ }^{1}$ Voir Ileana ChIRASSI COLOMBO, «Un pellegrinaggio del fantastico : itinerario al regno di Sibylla », Homo Viator, Atti del Convegno 18-19 ottobre 1996, Abbazia di Fiastra, Tolentino, 1997, pp. 37-64.
} 
1442, ce conte d'Antoine de La Sale ${ }^{2}$ constitue une partie d'un ouvrage plus vaste intitulé La Salade, sorte de compilation, dédiée à Jean de Calabre (fils aîné de René d'Anjou et héritier du roi de Sicile), de «mains livres» des auteurs classiques, que l'auteur compare à une salade où «se mettent pluiseurs bonnes herbes ». Comme l'écrit Danielle Régnier-Bohler, la réception de ce texte par un «milieu princier, lettré et préhumaniste », la cour d'Anjou-Provence, explique que « pour distraire ces nobles princes, le conte propose une savante évasion. $»^{3}$

En 1420, Antoine de La Sale a séjourné en Italie, et a peut-être visité la grotte de la Sibylle à Montemonaco, dans la région d'Ancône, où survivait alors l'étrange rumeur. C'est donc en connaisseur de la géographie de la péninsule (il y a séjourné de nombreuses fois) qu'Antoine décrit les différentes parties réelles du pays, mais également ce pays fabuleux qu'est le Paradis de la reine Sibylle.

L'œuvre se fonde sur un espace fortement conditionné par le clivage entre la liste varronienne des dix vraies sibylles et la créature qui porte le nom de Sebile. Le prologue, l'épilogue et les croquis instaurent une opposition linguistique et iconographique qui marque la place privilégiée d'où émane le discours. L'espace littéraire se trouve divisé, tiraillé entre le centre et la marge, et la sibylle est répertoriée tantôt dans le domaine des choses véritables et certaines, tantôt dans celui des choses étranges, collectées pour le divertissement des princes ou pour le simple plaisir de raconter ${ }^{4}$.

\footnotetext{
${ }^{2}$ Antolne De La SALE, Le Paradis de la reine Sibylle, édition et commentaire critique par Fernand Desonay, Paris, Droz, 1930. F. Desonay édite le manuscrit de Chantilly, conservé au musée Condé (ms 653). Sur l'écrivain, voir le récent ouvrage de Sylvie LEFEVRE, Antoine de La Sale, La fabrique de l'œuvre et de l'écrivain, suivi de l'édition critique du Traité des Anciens et Nouveaux Tournois, Genève, Droz, Publications Romanes et Françaises, 2006.

${ }^{3}$ Voir Danielle Bohler-Regnier, « La Sibylle dans la Salade d'Antoine de La Sale. Enquête sur l'imaginaire de la figure séductrice et satanique au $X V^{\mathrm{e}}$ siècle en milieu princier » dans Ileana ChIRASSi Colombo et Tullio SePPILli (éds), Sibille e Linguaggi oracolari. Mito, storia, tradizione, 1999, pp. 673-694; ici, p. 678.

${ }^{4} \mathrm{Je}$ ne me préoccupe pas ici du lien entre la prophétesse de l'Antiquité et la fée médiévale. Voir pour cette reconstitution deux travaux de Francine MORA-LEBRUN : "Postface ", dans Antoine De La Sale, Le Paradis de la reine Sibylle, Paris, Stock/Moyen Age, 1983, et « Métamorphoses dans le Paradis de la reine Sibylle d'Antoine de La Sale : des archétypes aux jeux d'une écriture ", dans Laurence HARF-LANCNER (éd), Métamorphose et bestiaire
} 
Je me propose de relire le conte d'Antoine de La Sale en montrant ce qu'a de problématique cette double existence de la sibylle. Le texte invente une polyvalence de la prophétesse en faisant se côtoyer l'ambigu et l'hétérogène propre aux contes à l'univoque et l'homogène de la tradition didactique ou religieuse. Je vais essayer de montrer comment ce texte manipule la notion de marge à trois niveaux : un niveau géographique, qui insère le pays imaginaire dans le réel tout en marquant une frontière ; un niveau générique, qui confronte l'écriture encyclopédique à l'écriture de l'imaginaire; et un niveau textuel, qui inverse les valeurs en séparant les espaces extrêmes du prologue et de l'épilogue, consacrés à l'acte d'énonciation et aux valeurs communes, et l'espace central, où se déploie la fable du Paradis.

Le récit d'Antoine est une excursion dans un pays insolite qui acquiert une réalité physique pour le lecteur : le mont de la Sibylle. Le gouverneur qui relate une fiction où l'imaginaire pur se mêle à une réinvention de la géographie franchit allègrement la barrière séparant les simples notes de voyage personnel et le voyage intérieur.

Le pays où se trouve l'étrange paradis est une marge géographique : «le mont est es parties de la marque d'Enconne et ou terrouer d'un chastel nommé Montemoynaco, qui est autant a dire comme le mont du moyne $»^{5}$. La verticalité de la montagne redouble cet effet de marge : l'entrée de la grotte se trouve au sommet du mont, à treize kilomètres de la ville. Par la suite, le texte est saturé par la multiplication des marqueurs de frontière ou de séparation (le pont, les distances, etc).

Le paysage décrit, celui de la montagne, apparaît à bien des égards comme un lieu d'effroi : le mont, aride et rocailleux, abrite des curiosités

fantastique au Moyen Âge, Paris, Collection de l'ENS de Jeunes Filles n²8, 1985, pp. 291300. On peut lire aussi William L. KinTER et Joseph R. KeLler (éds), The Sibyl: Prophetess of Antiquity and Medieval Fay, Philadelphie, 1968.

${ }^{5}$ Voir Le Paradis de la reine Sibylle, éd. F. Desonay, p. 9. 
végétales, le «pollibastro » et la «chentofollie ${ }^{6}$. Le narrateur décrit un spectacle de haute montagne : "et sans vent fait il grant hideur a veoir la vallee de tous costez, et souverainement a la main dextre; car elle est si treshideuse de

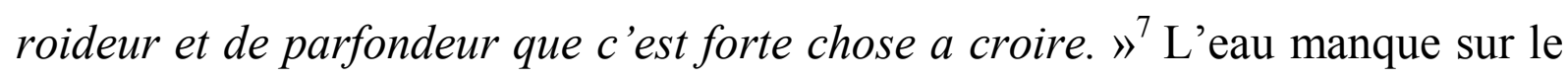
chemin de cette excursion qui ressemble plutôt à une expédition spéléologique : excentrement par rapport a monde connu, humain et rassurant.

L'organisation de l'espace fait que, de loin en loin, on repère grâce au voyage quelques jalons qui émergent. La toponymie se veut pittoresque, garantissant l'authenticité, les indications topographiques sont très précises. La merveille autochtone est mise en œuvre pour ébranler les repères anthropologiques et spatiaux familiers à l'esprit occidental du $\mathrm{XV}^{\mathrm{e}}$ siècle, d'où la tension permanente qui est créée entre les images de l'espace référentiel (le « centre », l'univers d'ici impliqué dans l'acte d'énonciation) et les images de la marge, dissimulée dans un pli de la terre et peuplée d'une sibylle fantastique.

L'entrée dans la grotte établit un deuxième niveau de marginalité. La sibylle n'est pas visible: "la est l'entree de la cave a main droitte; et est l'entree petite, en forme d'un escu, aguë dessus et large dessoubs. E y a une roche au devant; et celui qui y veult entrer, il lui convient tresfort baissier et entrer a quatre piez, descendant les piés contre bas jusques a une chambrette toute quarree $»^{8}$. Au fond se trouve un trou rond, de la taille d'une tête d'homme, qui ne donne que bien peu de lumière. Les connotations nocturnes, l'atmosphère mystérieuse, les sèmes d'inversion, la difficulté due à l'étroitesse du passage dans l'autre monde, tout incite à faire de ce paradis un royaume à la marge : « il convient descendre les piez premiers, car autrement nul aller n'y pourroit, tant est la cave estroitte et petite en descendant fort contre bas ${ }^{9}$. Cette marge

\footnotetext{
${ }^{6}$ Ibid., pp. 10-11.

${ }^{7}$ Ibid., p. 13.

${ }^{8}$ Ibid., p. 14.

${ }^{9}$ Ibid., p. 14.
} 
souterraine, hors de la vue de tous, s'ouvre comme un espace inverse, vecteur de prodige.

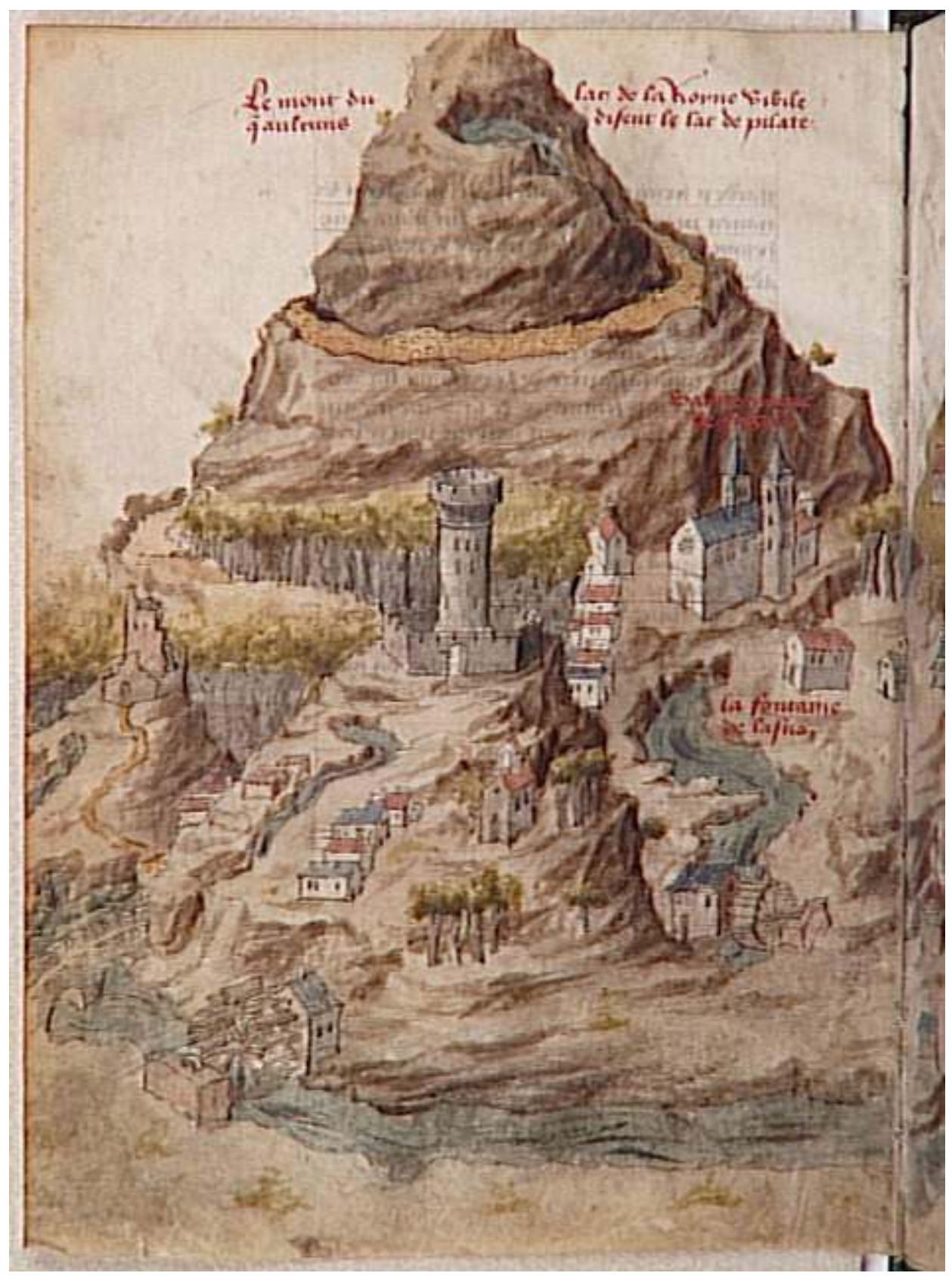

ci-dessous et ci-contre : les « pourtraicts » des monts décrits par Antoine, tels qu'on les trouve dans le ms 653 du musée Condé de Chantilly 
Le paradis de la sibylle est ainsi suspendu entre le probable et l'incroyable. La marge géographique donne au voyage et au récit la valeur d'un défi, l'allure d'une transgression. La pénétration de cet univers « hérétique »,

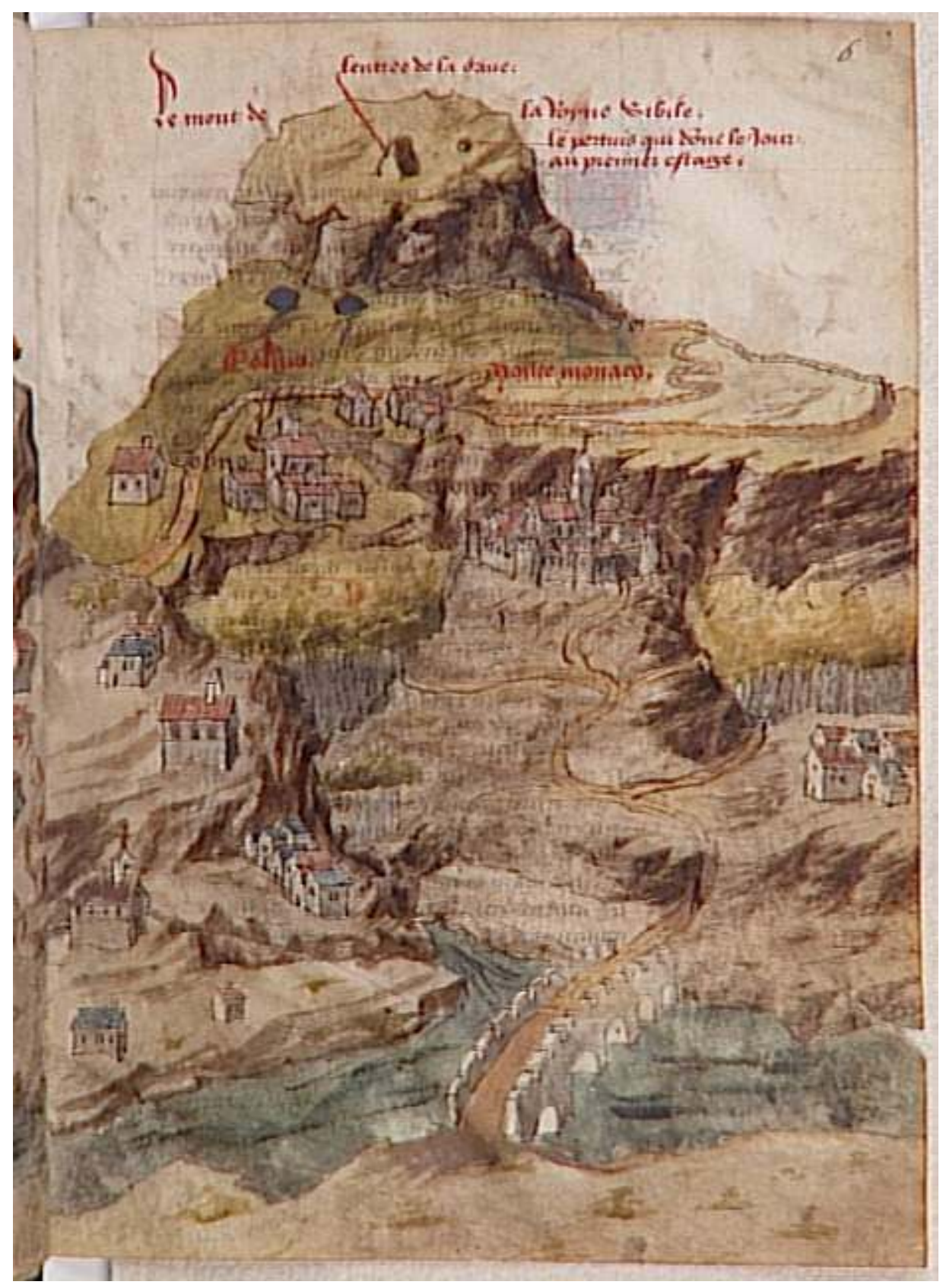

qui se situe hors de l'orthodoxie sibylline, vaut, pour deux voyageurs anciens qu'Antoine évoque au passage, une excommunication : à trop vouloir pénétrer les marges morganiennes du monde, on s'exclut de celui-ci. Le récit met en place deux mondes qui dissonent: le didactisme scientifique confronte aux 
rumeurs étranges rapportées de l'expédition la langue discordante de l'autorité papale qui émet l'interdit, énonce ce qu'il faut croire et ne pas croire, instaure des frontières fixes entre centre et marge. Le récit d'Antoine de La Sale réserve deux traitements de l'imaginaire de la sibylle: l'un normatif, l'autre chimérique.

L'insistance sur la frontière entre le monde connu et sa marge, inscrite dans sa géographie mais habilement dissimulée aux regards, fait du Paradis de la reine Sibylle un récit à propos duquel on n'ose pas ou ne peut pas décider du possible et de l'impossible. La marge géographique établit une marge générique ; l'imaginaire des terres incertaines a la bride sur le cou.

Le récit permet une échappée vers le monde des merveilles, où l'illusion d'optique remplace l'effet de réel. L'objet de prédilection du récit médiéval, on le sait, est l'exploration de régions inexplorées, d'espaces périlleux protégés par un tabou : les narrateurs y épousent les frontières pour en explorer les marges, à l'image des chevaliers qui les affrontent pour les faire reculer. Au-delà de la frontière, là où gît le monstre, la male chose, les repères s'abolissent selon un thème médiéval bien connu : tout être qui y entre subit une temporalité brouillée, une spatialité mythique. Dans le Perceforest, le personnage nommé Sebile a le pouvoir de faire oublier le temps ${ }^{10}$. Dans le récit d'Antoine, la reine Sebile connaît toutes les langues et son royaume incarne une Babel inversée.

D'un point de vue générique, c'est tout le monde des rêveries romanesques et des jeux littéraires qui s'ouvre alors : le texte établit une riche intertextualité avec Calypso (recherche du père italien, qui aurait séjourné chez une nymphe), avec Enée (même recherche du lignage, auprès de la sage sibylle de Cumes), etc. Cependant, l'imagination ne s'assume pas vraiment, elle ne se

\footnotetext{
${ }^{10}$ Voir Le roman de Perceforest, début de la première partie, éd. Jane H. M. TAYLOR, Genève, Droz (TLF), 1979, 11. 6089-8184, pp. 235-294, et l'étude de Christine ACHERFERLAMPIN, «Sebille prophétesse et maternelle : du monde antique au monde arthurien dans Perceforest ", dans La Sibylle, parole et représentation, Presses universitaires de Rennes, collection «Interférences », 2004, pp. 211-225.
} 
conçoit que comme un autre possible, fantasmé, de l'histoire officielle. Cette marge est l'espace où surgit non une vérité, mais la rumeur : "Et pour ce que en icelle cave a pluiseurs estranges et merveilleuses choses, si comme est le commun parler des gens, non mye pour chose qui evidamment se puisse tesmoigner, fors de tant que encores me fut compté par pluiseurs gens d'eglise et autres (...) $»^{11}$. De fait, tout ce qu'Antoine rapporte de ce pays est «selon le dit des gens du pays » ${ }^{12}$ : 1'expression revient tout au long du texte.

De la même façon qu'il n'est pas allé très loin dans son voyage d'exploration, Antoine de La Sale refuse d'aller plus loin dans sa contestation de la tradition. Pénétrer cet univers de la marge, au sens moral comme au sens spatial, serait commettre un outrage, s'exposer au mal, et en venir à se faire le complice de ce mal. La créature se colore alors de réprobation chrétienne, dans un texte qui finit par prendre ses distances avec sa propre audace.

Antoine construit son œuvre de façon à bien mettre en évidence, dans le prologue et l'épilogue de son récit, son credo. Il souligne la forte ambivalence qui sépare les vraies sibylles et «ceste faulse Sibille que le deable, par son povoir, a cause de nostre foible creance, a mis la renomee sus pour decevoir les simples gens. ${ }^{13}$ Antoine de La Sale entend démasquer une superstition, comme tous les polygraphes médiévaux qui ont enregistré cette légende sulfureuse :

« La Sibylle de la caverne n'est qu'une «faulse Sibille », dont la duplicité anthroponymique renvoie à l'obsession médiévale du simulacre et des tests d'identité. Dans la tradition de doublets redoutables, la fausse Sibylle, tout comme la fausse Guenièvre, trompe les naïfs $\gg{ }^{14}$.

C'est très clair au début du Paradis :

\footnotetext{
${ }^{11}$ Voir Le Paradis de la reine Sibylle, éd. F. DESONAY, p. 17.

12 Ibid., p. 7.

${ }^{13}$ Ibid., p. 55.

${ }^{14}$ Danielle Bohler-Regnier, «La Sibylle dans la Salade d'Antoine de La Sale. », art. cit., p. 688.
} 
«Mon tresredoubté seigneur, apprès les ystoires cy devant dictes, pour rire et passer temps, vous escrips les merveilleuses choses que sont es mons de la Sibile et de son lac. ${ }^{15}$

Au cœur d'un exposé géographique très sérieux donc, un espace pour le rire et le « passe-temps ».

Cette distance est aussi sensible à la fin, puisqu'Antoine en vient à rayer le texte précédent en l'accusant de fausseté et en citant les autorités. Le thème sibyllin est repris, mais cette fois dans son aspect sérieux, canonique :

"Si prie a Dieu qu'[il] gart chascun bon crestien de celle faulse creance et de soy mectre en ce peril. Lesquelles choses, pour rire et passer temps, pour montrer a chascun que le contraire, j'ay mis tout en escript. (...) Que, quant vostre plaisir et de ma redoubtee dame de Calabre, vostre compaigne sera de y aller pour vous esbactre, disant voz heures, en attendant l'eure du disner ou du soupper, ladicte royne et toutes ses dames a tresgrant joye vous y festoieront; et, oultre ce, y porrez acquerir tresgrans pardons et indulgences, qui vous metteront tous et toutes vestues en paradiz, tout aussi droict que une faussille. ${ }^{16}$

Pour Antoine, celui qui croit à tous ces mensonges n'a qu'une fausse opinion et une fausse croyance, et quitte le chemin de la vérité. Aussi s'en réfère-t-il au texte qui représente la voie droite et la correction de toutes les voies déviantes : les Saintes Ecritures. D’abord parce que la Passion du Christ est censée avoir mis fin à toutes les formes de diablerie. Ensuite parce qu'un grand nombre de textes s'accordent pour dire qu'il n'a existé que dix sibylles. Et il cite saint Augustin, qui cite Lactance, qui cite Varron.

Le manuscrit 653 du musée Condé (enluminé vers 1440) ${ }^{17}$ confronte un e série d'images de la fausse sibylle avec l'illustration unique de la légende de 1'Ara coeli, où la sibylle Tiburtine montre à Auguste un soleil où apparaît la

\footnotetext{
${ }^{15}$ Voir Le Paradis de la reine Sibylle, éd. F. DesOnAY, p. 3.

${ }^{16}$ Ibid., pp. 55-56.

${ }^{17}$ Voir la notice de Sylvie LEFÈVRE dans L'enluminure en France au temps de Jean Fouquet, Somogy, 2003, p. 89. On admirera, dans le même volume, six sibylles annonçant la naissance du Christ, variation sur le thème de l'Ara coeli, extraites des Heures d'Adélaïde de Savoie, p. 39.
} 
Vierge Marie, tenant dans son giron l'enfant Jésus. Rappel ultime du dogme, grâce à une légende souvent reprise dans l'iconographie de la fin du Moyen Age : elle est annoncée par une rubrique dans le manuscrit (« Comment la Sibile Turbitina [sic] monstra la vierge et son enfant qui seroit Dieu et nul aultre a Octavien Auguste qui se vouloit faire adorer comme Dieu $\rangle^{18}$ ); pourtant, il n'en est pas question dans le texte d'Antoine de La Sale. La sibylle retrouve sa vraie place : à la marge du texte, elle vient redire quelles sont les valeurs centrales de la cité. Le tourbillon des références (Gracius, monseigneur saint Augustin, Lactance, Isidore, Martin), associé à l'autorité de l'image, viennent biffer tout le récit précédent.

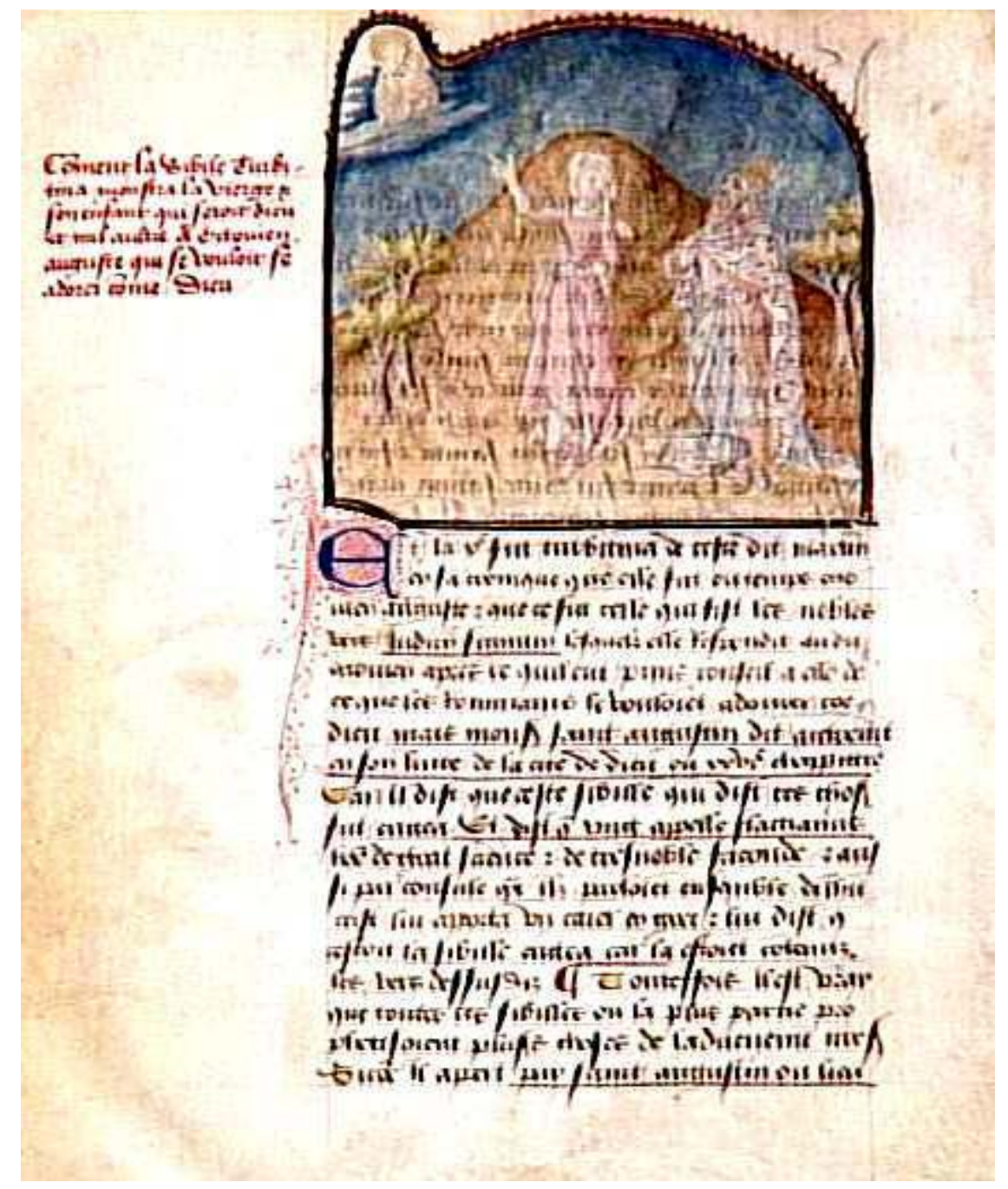

${ }^{18}$ Voir Le Paradis de la reine Sibylle, éd. F. DeSONAY, p. 54. 
Avec ce paradis, tout s'inscrit dans l'affrontement ludique d'un centre et d'une marge pour l'instauration d'une vérité sur les sibylles. Cette tension, qui exprime un choix religieux et esthétique, est aussi convertie en termes géographiques, esthétiques, génériques, d'où les multiples jeux d'expulsion, de rejet et d'exclusion de la faussaire. Finalement, la vérité triomphe, donnant la pleine maîtrise de l'espace à la liste de Varron, moins vulnérable, qui ne s'enrichit pas de cette aventure de traverse, contrairement à ce qui se passait dans l'Antiquité, où la découverte de sibylles nouvelles était un fait constant. La finalité didactique est préservée : en se concentrant sur la marge, on montre paradoxalement qu'il y a un centre essentiel à garder, voire à reconquérir sur la rumeur. Le centre : la longue tradition savante qui fixe les grands traits de la prophétesse. La marge : le lieu mental où s'abolit le conventionnel et s'exténue l'exigence de vérité, le lieu du colportage, de la rumeur intégrant fables et peurs. Antoine joue la marge contre le centre, la rumeur contre le canon, l'expérience contre l'historicité.

L'imaginaire pur est effrayant parce qu'il engendre des monstres, nous disent les poètes. Mais il se double ici d'une relecture par la raison qui en fixe les limites, pour reléguer l'insolite dans les sphères de l'inexistant: l'œuvre d'Antoine de La Sale prend la forme d'une sortie (temporaire) vers l'imagination suivie d'un retour à la normalité. L'auteur donne toute sa force et son sens au spectacle de l'imagination en le reléguant in extremis aux marges, comme si cela était pour lui un amusement, un rêve.

Le renversement final est profondément ambivalent, car il fait disparaître ce lieu où le rire et la peur pouvait éclater sans retenue ni censure. L'œuvre semble ainsi combiner, «par sa puissance parodique, la mort et la résurrection, 
la négation (ironie) et l'affirmation (rire de jubilation) » ${ }^{19}$. Elle se construit comme un ensemble hétéroclite, d'où l'ouverture ironique du finale, qui indique la distance prise par le récit à l'égard de la convention même de la fiction. Les phrases qui closent le récit ramènent le lecteur au début du texte, où l'aventure n'était présentée que comme une rumeur fausse. Ironique circularité, où la marge prend la place du centre et vice versa.

Le matériau double préexistait, et la normalisation était attendue. C'est l'agencement qui est inédit, et ce « collage» présenté finit par nous convaincre que toute littérature est de seconde main.

${ }^{19}$ Mikhaïl BAKHTINE, Esthétique et théorie du roman, traduit du russe par Daria OLIVIER, Paris, Gallimard, coll. Bibliothèque des Idées, 1978. 